\title{
SOME FLATNESS CONDITIONS ON NORMAL METRIC CONTACT PAIRS
}

\author{
İnan ÜNAL \\ Department of Computer Engineering, Munzur University, Tunceli, TURKEY
}

\begin{abstract}
In this paper, the geometry of normal metric contact pair manifolds is studied under the flatness of conformal, concircular and quasi-conformal curvature tensors. It is proved that a conformal flat normal metric contact pair manifold is an Einstein manifold with a positive scalar curvature and has positive sectional curvature. It is also shown that a concircular flat normal metric contact pair manifold is an Einstein manifold. Finally, it is obtained that a quasi-conformally flat normal metric contact pair manifold is an Einstein manifold with a positive scalar curvature and, is a space of constant curvature.
\end{abstract}

\section{INTRODUCTION}

Contact transformations were defined as a geometric tool to study system of differential equations in 1872 by S.Lie [1]. Afterward the notion of contact manifolds has occurred in the manifold theory. Contact manifolds have many applications in mathematics and some applied areas such as mechanics, optics, thermodynamics, control theory and theoretical physics 2]. The Riemannian geometry of contact manifolds give us some geometric interpretation about Einstein manifolds which are arisen from the theory of relativity.

A conformal transformation is a map which converts a metric to another with preserving angle between two vector fields. Conformal curvature tensor on a Riemann manifold is a curvature tensor of the $(1,3)$-type that is invariant under conformal transformations. This tensor gives important information about the Riemann geometry of the manifold. If it vanishes then the manifold is said to be conformally flat, that's mean the manifold is flat under conformal transformations. A concircular transformation is a special conformal transformation and, preserves the geodesic circle. These type of transformations and their applications to differential geometry

2020 Mathematics Subject Classification. Primary 53C15, 53C25; Secondary 53D10.

Keywords and phrases. Contact pairs, curvature tensor, bicontact.

¿ inanunal@munzur.edu.tr

(D) 0000-0003-1318-9685.

(C) 2020 Ankara University Communications Faculty of Sciences University of Ankara-Series A1 Mathematics and Statistics 
were studied by Yano [3]. In same paper Yano defined concircular curvature tensor and showed that this tensor is invariant under concircular transformations. A manifold is called concircularly flat if this tensor vanishes. Yano and Sawaski 4 introduced quasi-conformal curvature which includes both concircular and conformal curvature tensor as special cases. If this tensor vanishes on the manifold identically then the manifold is called quasi-conformally flat. Flatness conditions of conformal, concircular and quasi-conformal curvature tensors on contact manifolds has many geometric and physical applications. For example, while a conformal flat Sasakian manifold is of constant curvature [5], a normal complex contact metric manifold is not conformal, concircular and quasi-conformal flat [6].

Blair, Ludden and Yano [7] studied on complex manifolds consider the results on Calabi-Eckman manifolds $S^{2 p+1} \times S^{2 q+1}$. By consider two Sasakian structure on $S^{2 p+1}$ and $S^{2 q+1}$ they gave the second fundamental form on Calabi-Eckman manifold, defined Hermitian bicontact manifold and obtained an $f$-structure on bicontact manifolds. Also normality of bicontact manifolds was given in same work. Bande and Hadjar 8 studied on bicontact manifolds under the name contact pairs. Further they considered a special type of $f$-structure with complementary frames related to a contact pair and, called by contact pair structure. The normality of contact pair structures were given by same authors 9 11. The conformal flatness of a normal metric contact pair manifold were studied by Bande, Blair and Hadjar 12]. They proved that a conformal flat normal metric contact pair manifold is locally isometric to Hopf manifold $S^{2 p+1}(1) \times S^{1}$.

In this paper we studied on conformal, concircular and quasi-conformal curvature flatness of normal metric contact pair manifold. We prove that a conformal flat normal metric contact pair manifold is an Einstein manifold with a positive scalar curvature and, has positive sectional curvature. Also we obtain that a concircular flat normal metric contact pair manifold is an Einstein manifold. Finally we prove that a quasi-conformal flat normal metric contact pair manifold is an Einstein manifold with a positive scalar curvature and, is a space of constant curvature.

\section{Preliminaries}

In this section a short survey is given for contact manifolds and contact pair structures. For detail we refer to reader $8,10,13$.

2.1. Real and Complex Contact Manifolds. A real contact manifold is defined by a contact form $\eta$ which is a volume form on a real $(2 p+1)-$ dimensional differentiable manifold $M$. The kernel of $\eta$ defines $2 p$-dimensional a non-integrable distribution of $T M$ :

$$
\mathcal{D}=\{X: \eta(X)=0, X \in \Gamma(T M)\} .
$$

We also recall $\mathcal{D}$ contact or horizontal distribution. Let take a vector field $\xi$ on $M$ which is dual vector of $\eta$. Then for $(1,1)$-tensor field $\varphi, M$ is called an almost 
contact metric manifold if following conditions are satisfied:

$$
\varphi^{2}=-I+\eta \otimes \xi, \quad \eta(\xi)=1, \quad g(\varphi \bullet, \bullet)=-g(\bullet, \varphi \bullet)
$$

where $I$ is identity map on $T M$ and $g$ is a Riemannian metric [13]. Also we call $g$ by compatible metric. Similar to Kähler manifold we have a second fundamental form on an almost contact metric manifold $\Omega(\bullet, \bullet)=d \eta(\bullet, \bullet)$. Also $d \eta(\bullet, \bullet)=g(\bullet, \varphi \bullet)$ and in this case we recall $g$ is an associated metric.

The geometry of contact manifold is studied in different classes. One of them is Sasakian manifold which has a Kähler form on Riemannian cone $M \times \mathbb{R}^{+}$. A Sasakian manifold has also an almost contact metric structure. The almost contact structure on a Sasakian manifold is normal i.e. $N(\varphi \bullet, \varphi \bullet)+2 d \eta(\bullet, \bullet) \xi=0$ where $N(\varphi \bullet, \varphi \bullet)$ is the Nijenhuis tensor field of $\varphi$.

In 1959 Kobayashi 14] defined complex analogue of a real contact manifold. Therefore the concept of complex contact manifold entered to the literature. 1980s Ishihara and Konishi [15] constructed almost contact structure on a complex contact manifold and they defined compatible metric. A complex almost contact metric manifold is a complex odd $(2 p+1)$-dimensional complex manifold with $(J, \varphi, \varphi \circ$ $J, \xi,-J \circ \xi, \eta, \eta \circ J, g)$ structure such that

$$
\begin{gathered}
\varphi^{2}=(\varphi J)^{2}=-I+\eta \otimes \xi-(\eta \circ J) \otimes(J \circ \xi), \\
\eta(\xi)=1, \eta(-J \circ \xi)=0,(\eta \circ J)(-J \circ \xi)=1,(\eta \circ J)(\xi)=0, \\
g(\varphi \bullet, \bullet)=-g(\bullet, \varphi \bullet), \quad g((\varphi \circ J) \bullet \bullet)=-g(\bullet,(\varphi \circ J) \bullet)
\end{gathered}
$$

where $g$ is a Hermitian metric on $M, J$ is a natural almost complex structure. The normality of complex almost contact metric manifolds were given by IshiharaKonishi and Korkmaz 15, 16. Normal complex contact metric manifolds were studied by several authors $6,16,17]$.

\subsection{Metric contact pair manifold.}

Definition 1. Let $M$ be a $(2 p+2 q+2)$-dimensional differentiable manifold. A pair of $\left(\alpha_{1}, \alpha_{2}\right)$ on $M$ is said to be a contact pair of type $(p, q)$ if

- $\alpha_{1} \wedge\left(d \alpha_{1}\right)^{p} \wedge \alpha_{2} \wedge\left(d \alpha_{2}\right)^{q} \neq 0$

- $\left(d \alpha_{1}\right)^{p+1}=0$ and $\left(d \alpha_{2}\right)^{q+1}=0$

where $p, q$ are positive integers [8].

For 1 -forms $\alpha_{1}$ and $\alpha_{2}$ we have two integrable subbundles of $T M ; \mathcal{D}_{1}=\{X$ : $\left.\alpha_{1}(X)=0, X \in \Gamma(T M)\right\}$ and $\mathcal{D}_{2}=\left\{X: \alpha_{2}(X)=0, X \in \Gamma(T M)\right\}$. Then we have two characteristic foliations of $M$, denoted by $\mathcal{F}_{1}=\mathcal{D}_{1} \cap \operatorname{kerd} \alpha_{1}$ and $\mathcal{F}_{2}=\mathcal{D}_{2} \cap$ kerd $\alpha_{2}$, respectively. $\mathcal{F}_{1}$ and $\mathcal{F}_{2}$ are $(2 p+1)$ and $(2 q+1)$-dimensional contact manifolds with contact form induced by $\alpha_{2}$ and $\alpha_{1}$ respectively. For a contact pair $\left(\alpha_{1}, \alpha_{2}\right)$ of type $(p, q)$ there are associated two commuting vector fields $Z_{1}$ and $Z_{2}$, called Reeb vector fields of the pair, which are determined uniquely by the following equations:

$$
\alpha_{1}\left(Z_{1}\right)=\alpha_{2}\left(Z_{2}\right)=1, \alpha_{1}\left(Z_{2}\right)=\alpha_{2}\left(Z_{1}\right)=0,
$$




$$
i_{Z_{1}} d \alpha_{1}=i_{Z_{1}} d \alpha_{2}=i_{Z_{2}} d \alpha_{1}=i_{Z_{2}} d \alpha_{2}=0
$$

where $i_{X}$ is the contraction with the vector field $\mathrm{X}$. In particular, since the Reeb vector fields commute, they determine a locally free $\mathbb{R}^{2}$-action, called the Reeb action.

The tangent bundle of $\left(M,\left(\alpha_{1}, \alpha_{2}\right)\right)$ can be decomposable by different ways. For the two subbundle of $T M$ which are given by

$$
T \mathcal{G}_{i}=k e r d \alpha_{i} \cap \operatorname{ker} \alpha_{1} \cap \operatorname{ker} \alpha_{2}, i=1,2,
$$

we can write

$$
T \mathcal{F}_{1}=T \mathcal{G}_{1} \oplus \mathbb{R} Z_{2} \text { and } T \mathcal{F}_{2}=T \mathcal{G}_{2} \oplus \mathbb{R} Z_{1}
$$

Therefore we get $T M=T \mathcal{G}_{1} \oplus T \mathcal{G}_{2} \oplus \mathbb{R} Z_{1} \oplus \mathbb{R} Z_{2}$. Also we can state $T M=\mathcal{H} \oplus \mathcal{V}$ for $\mathcal{H}=T \mathcal{G}_{1} \oplus T \mathcal{G}_{2}$ and $\mathcal{V}=\mathbb{R} Z_{1} \oplus \mathbb{R} Z_{2}$, we call $\mathcal{H}$ is horizontal subbundle and $\mathcal{V}$ is vertical subbundle of $T M$.

Let $X$ be an arbitrary vector field on $M$. We can write $X=X^{\mathcal{H}}+X^{\mathcal{V}}$, where $X^{\mathcal{H}}, X^{\mathcal{V}}$ horizontal and vertical component of $X$ respectively. For $X^{1} \in T \mathcal{F}_{1}$ and $X^{2} \in T \mathcal{F}_{2}$ we have $X=X^{1}+X^{2}$. Also we can write $X^{1}=X^{1^{h}}+\alpha_{2}\left(X^{1}\right) Z_{2}$ and $X^{2}=X^{2^{h}}+\alpha_{1}\left(X^{2}\right) Z_{1}$, where $X^{1^{h}}$ and $X^{2^{h}}$ are horizontal parts of $X^{1}, X^{2}$ respectively. Thus we have $X^{\mathcal{H}}=X^{1^{h}}+X^{2^{h}}, X^{\mathcal{V}}=\alpha_{1}\left(X^{2}\right) Z_{1}+\alpha_{2}\left(X^{1}\right) Z_{2}$. From all these decompositions of $X$ finally we get

$$
\begin{gathered}
X=X^{1^{h}}+X^{2^{h}}+\alpha_{1}\left(X^{2}\right) Z_{1}+\alpha_{2}\left(X^{1}\right) Z_{2}, \\
\alpha_{1}\left(X^{1^{h}}\right)=\alpha_{1}\left(X^{2^{h}}\right)=0, \quad \alpha_{2}\left(X^{1^{h}}\right)=\alpha_{2}\left(X^{2^{h}}\right)=0 .
\end{gathered}
$$

Since we have two different 1 -forms by above decomposition we understand the components of $X \in \Gamma(T M)$ in which distributions.

Definition 2. An almost contact pair structure on a $(2 p+2 q+2)$-dimensional manifold $M$ is a structure $\left(\alpha_{1}, \alpha_{2}, \phi, Z_{1}, Z_{2}\right)$, where $\left(\alpha_{1}, \alpha_{2}\right)$ is a contact pair, $\phi$ is $a(1,1)$ tensor field on $M$ and $Z_{1}, Z_{2}$ are Reeb vector fields such that:

$$
\phi^{2}=-I+\alpha_{1} \otimes Z_{1}+\alpha_{2} \otimes Z_{2}, \quad \phi Z_{1}=\phi Z_{2}=0 .
$$

The rank of $\phi$ is $(2 p+2 q)$ and $\alpha_{i}(\phi)=0$ for $i=1,2$.

The endomorphism $\phi$ is said to be decomposable if $T \mathcal{F}_{i}$ is invariant under $\phi$. If $\phi$ is decomposable then we have almost contact structure on $T \mathcal{F}_{i}$ for $i=1,2$ are induced from $\left(\alpha_{i}, Z_{i}, \phi\right), i \neq j, 10$. Unless otherwise stated we assume that $\phi$ is decomposable.

Definition 3. Let $\left(\alpha_{1}, \alpha_{2}, Z_{1}, Z_{2}, \phi\right)$ be an almost contact pair structure on a manifold $M$. A Riemannian metric $g$ is called

(1) compatible if $g\left(\phi X_{1}, \phi X_{2}\right)=g\left(X_{1}, X_{2}\right)-\alpha_{1}\left(X_{1}\right) \alpha_{1}\left(X_{2}\right)-\alpha_{2}\left(X_{1}\right) \alpha_{2}\left(X_{2}\right)$ for all $X_{1}, X_{2} \in \Gamma(T M)$.

(2) associated if $g\left(X_{1}, \phi X_{2}\right)=\left(d \alpha_{1}+d \alpha_{2}\right)\left(X_{1}, X_{2}\right)$ and $g\left(X_{1}, Z_{i}\right)=\alpha_{i}\left(X_{1}\right)$, for $i=1,2$ and for all $X_{1}, X_{2} \in \Gamma(T M)$ [10]. 
We call $\left(M, \phi, Z_{1}, Z_{2}, \alpha_{1}, \alpha_{2}, g\right)$ by a metric almost contact pair manifold and we have the following properties on $M$ [10]:

$$
\begin{aligned}
g\left(Z_{i}, X\right) & =\alpha_{i}(X), \quad g\left(Z_{i}, Z_{j}\right)=\delta_{i j}, \\
\nabla_{Z_{i}} Z_{j}=0, \nabla_{Z_{i}} \phi & =0
\end{aligned}
$$

and for every $X$ tangent to $M, i=1,2$ we have

$$
\nabla_{X} Z_{1}=-\phi_{1} X, \quad \nabla_{X} Z_{2}=-\phi_{2} X
$$

where $\phi=\phi_{1}+\phi_{2}[10$.

We have two almost complex structures on $\left(M, \phi, Z_{1}, Z_{2}, \alpha_{1}, \alpha_{2}, g\right)$ as;

$$
J=\phi-\alpha_{2} \otimes Z_{1}+\alpha_{1} \otimes Z_{2}, \quad T=\phi+\alpha_{2} \otimes Z_{1}-\alpha_{1} \otimes Z_{2} .
$$

Definition 4. A metric contact pair manifold is said to be normal if $J$ and $T$ are integrable [9].

Theorem 5. Let ( $\left.M, \phi, Z_{1}, Z_{2}, \alpha_{1}, \alpha_{2}, g\right)$ be a normal metric contact pair manifold. Then we have

$$
g\left(\left(\nabla_{X_{1}} \phi\right) X_{2}, X_{3}\right)=\sum_{i=1}^{2}\left(d \alpha_{i}\left(\phi X_{2}, X_{1}\right) \alpha_{i}\left(X_{3}\right)-d \alpha_{i}\left(\phi X_{3}, X_{1}\right) \alpha_{i}\left(X_{2}\right)\right),
$$

where $X_{1}, X_{2}, X_{3}$ are arbitrary vector fields on $M$ [10].

On a normal metric contact pair manifold we have $\nabla_{X} Z=-\phi X$ for $X \in \Gamma(T M)$ and $Z=Z_{1}+Z_{2}$.

We can consider a natural question: could any metric contact pair structure be considered locally the product of two contact metric manifold? An example of metric contact pair were given in [18, which is not locally product of two contact metric manifold. So metric contact pair structure has some different properties from contact metric manifolds and their results will be useful interpretation for the geometry of contact and complex manifolds.

2.3. Curvature properties of normal metric contact pair manifolds. We use the following statements for the Riemann curvature;

$$
\begin{aligned}
& R\left(X_{1}, X_{2}\right) X_{3}=\nabla_{X_{1}} \nabla_{X_{2}} X_{3}-\nabla_{X_{2}} \nabla_{X_{1}} X_{3}-\nabla_{\left[X_{1}, X_{2}\right]} X_{3}, \\
& \mathcal{R}\left(X_{1}, X_{2}, X_{3}, X_{4}\right)=g\left(R\left(X_{1}, X_{2}\right) X_{3}, X_{4}\right)
\end{aligned}
$$

for all $X_{1}, X_{2}, X_{3}, X_{4} \in \Gamma(T M)$. Also the Ricci operator is defined by

$$
Q X=\sum_{i=1}^{\operatorname{dim}(M)} R\left(X, E_{i}\right) E_{i},
$$

and the Ricci curvature and scalar curvature are given by

$$
\operatorname{Ric}\left(X_{1}, X_{2}\right)=g\left(Q X_{1}, X_{2}\right),
$$




$$
\text { scal }=\sum_{i=1}^{\operatorname{dim}(M)} \operatorname{Ric}\left(E_{i}, E_{i}\right) .
$$

where $E_{i}, 1 \leq i \leq \operatorname{dim}(M)$ are orthonormal basis of $M$. Let $Z=Z_{1}+Z_{2}$; from Lemma 3 of 12 for $X_{1}, X_{2}, X_{3}$ on a normal metric contact pair manifold with decomposable $\phi$ we have

$$
\begin{aligned}
\mathcal{R}\left(X_{1}, Z, Z, X_{3}\right)= & d \alpha_{1}\left(\phi X_{3}, X_{1}\right) \alpha_{1}\left(X_{2}\right)+d \alpha_{2}\left(\phi X_{3}, X_{1}\right) \alpha_{2}\left(X_{2}\right) \\
& -d \alpha_{1}\left(\phi X_{3}, X_{1}\right) \alpha_{1}\left(X_{2}\right)-d \alpha_{2}\left(\phi X_{3}, X_{2}\right) \alpha_{2}\left(X_{1}\right) .
\end{aligned}
$$

Thus for $X_{4}, X_{5} \in \Gamma(\mathcal{H})$ we get

$$
\begin{gathered}
R\left(X_{4}, Z, Z, X_{5}\right)=-g\left(X_{4}, X_{5}\right), \\
R\left(Z, X_{4}\right) X_{5}=d \alpha_{1}\left(\phi X_{5}, X_{4}\right) Z_{1}+d \alpha_{2}\left(\phi X_{5}, X_{4}\right) Z_{2}, \\
R\left(X_{1}, Z\right) Z=-\phi^{2} X_{1} .
\end{gathered}
$$

Let take an orthonormal basis of $M$

$\left\{E_{1}, E_{2}, \ldots, E_{p}, \phi E_{1}, \phi E_{2}, \ldots, \phi E_{p}, E_{p+1}, E_{p+2}, \ldots, E_{p+q}, \phi E_{p+1}, \phi E_{p+2}, \ldots, \phi E_{p+q}, Z_{1}, Z_{2}\right\}$ then for all $X_{1} \in \Gamma(T M)$ we get the Ricci curvature of $M$ as

$$
\operatorname{Ric}\left(X_{1}, Z\right)=\sum_{i=1}^{2 p+2 q}\left[d \alpha_{1}\left(\phi E_{i}, E_{i}\right) \alpha_{1}\left(X_{1}\right)+d \alpha_{2}\left(\phi E_{i}, E_{i}\right) \alpha_{2}\left(X_{1}\right)\right] .
$$

So, we obtain the following results:

$$
\begin{aligned}
& \operatorname{Ric}\left(X_{1}, Z\right)=0, \text { for } X_{1} \in \Gamma(\mathcal{H}), \\
& \operatorname{Ric}(Z, Z)=2 p+2 q, \\
& \operatorname{Ric}\left(Z_{1}, Z_{1}\right)=2 p, \operatorname{Ric}\left(Z_{2}, Z_{2}\right)=2 q, \operatorname{Ric}\left(Z_{1}, Z_{2}\right)=0 .
\end{aligned}
$$

Conformal $\mathcal{C}$, concircular $\mathcal{W}$ and quasi-conformal curvature tensor $\widetilde{\mathcal{C}}$ on a $(2 p+2 q+$ 2)-dimensional normal contact metric pair manifold are given by:

$$
\begin{aligned}
\mathcal{C}\left(X_{1}, X_{2}\right) X_{3}= & R\left(X_{1}, X_{2}\right) X_{3} \\
& +\frac{\text { scal }}{(2 p+2 q+1)(2 p+2 q)}\left(g\left(X_{2}, X_{3}\right) X_{1}-g\left(X_{1}, X_{3}\right) X_{2}\right) \\
& +\frac{1}{2 p+2 q}\left(g\left(X_{1}, X_{3}\right) Q X_{2}-g\left(X_{2}, X_{3}\right) Q X_{1}\right. \\
& \left.+\operatorname{Ric}\left(X_{1}, X_{3}\right) X_{2}-\operatorname{Ric}\left(X_{2}, X_{3}\right) X_{1}\right), \\
\mathcal{W}\left(X_{1}, X_{2}\right) X_{3}= & R\left(X_{1}, X_{2}\right) X_{3} \\
& -\frac{s c a l}{(2 p+2 q+2)(2 p+2 q+1)}\left[g\left(X_{2}, X_{3}\right) X_{1}-g\left(X_{1}, X_{3}\right) X_{2}\right] \\
\widetilde{\mathcal{C}}\left(X_{1}, X_{2}\right) X_{3}= & a R\left(X_{1}, X_{2}\right) X_{3}+b\left[\operatorname{Ric}\left(X_{2}, X_{3}\right) X_{1}-\operatorname{Ric}\left(X_{1}, X_{3}\right) X_{2}\right. \\
& \left.+g\left(X_{2}, X_{3}\right) Q X_{1}-g\left(X_{1}, X_{3}\right) Q X_{2}\right]
\end{aligned}
$$




$$
-\frac{s c a l}{2 p+2 q+2}\left[\frac{a}{2 p+2 q+1}+2 b\right]\left[g\left(X_{2}, X_{3}\right) X_{1}-g\left(X_{1}, X_{3}\right) X_{2}\right]
$$

where $X_{1}, X_{2}, X_{3} \in \Gamma(T M), a$ and $b$ are constants.

\section{Hermitian Contact Pair Manifolds}

In this section we give an almost contact pair structure on a Hermitian manifold.

Let $(M, g, J)$ be $(2 p+2 q+2)$-dimensional Hermitian manifold and $\left(\varphi_{1}, \eta_{1}, \xi_{1}\right)$, $\left(\varphi_{2}, \eta_{2}, \xi_{2}\right)$ be two almost contact structures on $M$ with following properties.

$$
\begin{aligned}
& g\left(\varphi_{i} X_{1}, X_{2}\right)=-g\left(X_{1}, \varphi_{i} X_{2}\right), \text { for } i=1,2, \\
& J \xi_{1}=-\xi_{2}, J \xi_{2}=\xi_{1}, \\
& \varphi_{i}^{2} X_{1}=-X_{1}+\eta_{1}\left(X_{1}\right) \xi_{1}+\eta_{2}\left(X_{1}\right) \xi_{2}, \\
& \varphi_{1}\left(J X_{1}\right)=-J \varphi_{1} X_{1}=\varphi_{2} X_{1}, \\
& \varphi_{2}\left(J X_{1}\right)=-J \varphi_{2} X_{1}=-\varphi_{1} X_{1}, \\
& \varphi_{2}\left(\varphi_{1} X_{1}\right)=-\varphi_{1}\left(\varphi_{2} X_{1}\right)=J X_{1}+\eta_{1}\left(X_{1}\right) \xi_{2}-\eta_{2}\left(X_{1}\right) \xi_{1}
\end{aligned}
$$

where $X_{1}, X_{2}$ are two arbitrary vector fields on $M[19$.

Let take $\phi=\varphi_{1} \circ \varphi_{2}$. Then $\phi$ is a $(1,1)$ tensor field on $M$. By direct computation we get

$$
\phi^{2} X_{1}=-X_{1}+\eta_{1}\left(X_{1}\right) \xi_{1}+\eta_{2}\left(X_{1}\right) \xi_{2} .
$$

Thus we obtain an almost contact pair structure on $M$ with the contact pair $\eta_{1}, \eta_{2}$ and we state:

Corollary 6. Let $\left(M^{2 p+2 q+2}, J, g\right)$ be an almost Hermitian manifold and $\left(\varphi_{i}, \eta_{i}, \xi_{i}\right)_{i=0}^{2}$ be two almost contact structure on $M$ with properties are given in (4). Then $\left(\eta_{1}, \eta_{2}, \phi\right)$ is an almost contact pair structure on $M$ such that

$$
\begin{gathered}
\phi^{2} X_{1}=-X_{1}+\eta_{1}\left(X_{1}\right) \xi_{1}+\eta_{2}\left(X_{1}\right) \xi_{2} \\
\eta_{i}\left(\xi_{j}\right)=\delta_{i j}, 1 \leq i, j, \leq 2, \\
\phi\left(\xi_{i}\right)=0
\end{gathered}
$$

for all $X_{1} \in \Gamma(T M)$.

Also for $X_{1}, X_{2} \in \Gamma(T M)$ we have

$$
g\left(\phi X_{1}, X_{2}\right)=-g\left(X_{1}, \phi X_{2}\right)
$$

and

$$
g\left(\phi X_{1}, \phi X_{2}\right)=g\left(X_{1}, X_{2}\right)-\eta_{1}\left(X_{1}\right) \eta_{1}\left(X_{2}\right)-\eta_{2}\left(X_{1}\right) \eta_{2}\left(X_{2}\right) .
$$

Thus we obtain compatible metric with contact pair structure.

These results show that a contact pair structure on an almost Hermitian manifold could be obtained from two almost contact structure on this manifold. Since contact pair manifolds have some significant properties, some future works could be done for Hermitian and contact structure. Also if the manifold is complex, then 
$\left(M, \varphi_{1}, \varphi_{2}, J, \xi_{1}, \xi_{2}, \eta_{1}, \eta_{2}, g\right)$ is a complex almost contact metric manifold. This type of manifolds were studied by several authors [6, 6 , 14 , 17$]$.

\section{Flatness Conditions on Normal Contact Pair Manifolds}

In this section we give some results on the flatness of conformal, concircular and quasi-conformal curvature tensors.

Theorem 7. A conformal flat normal metric contact pair manifold is an Einstein manifold with positive scalar curvature and has positive sectional curvature.

Proof. Let $\left(M, \phi, \alpha_{1}, \alpha_{2}\right)$ be a normal metric contact pair manifold. Suppose that $M$ is conformal flat. Then we have

$$
\begin{aligned}
\mathcal{R}\left(X_{1}, X_{2}, X_{3}, X_{4}\right)= & -A\left[g\left(X_{2}, X_{3}\right) g\left(X_{1}, X_{4}\right)-g\left(X_{1}, X_{3}\right) g\left(X_{2}, X_{4}\right)\right] \\
& -B\left(g\left(X_{1}, X_{3}\right) \operatorname{Ric}\left(X_{2}, X_{4}\right)-g\left(X_{2}, X_{3}\right) \operatorname{Ric}\left(X_{1}, X_{4}\right)\right. \\
& \left.+\operatorname{Ric}\left(X_{1}, X_{3}\right) g\left(X_{2}, X_{4}\right)-\operatorname{Ric}\left(X_{2}, X_{3}\right) g\left(X_{1}, X_{4}\right)\right),
\end{aligned}
$$

where $A=\frac{s c a l}{(2 p+2 q+1)(2 p+2 q)}$ and $B=\frac{1}{2 p+2 q}$. Taking $X_{2}=X_{3}=Z$ and $X_{1}, X_{4} \in$ $\Gamma(\mathcal{H})$ in (5), since $g(Z, Z)=2$ and from (1), we obtain

$$
\mathcal{R}\left(X_{1}, Z, Z, X_{4}\right)=-2 A g\left(X_{1}, X_{4}\right)-2 B \operatorname{Ric}\left(X_{1}, X_{4}\right)-(2 p+2 q) g\left(X_{1}, X_{4}\right) .
$$

Also from (1) we get

$$
2 \operatorname{BRic}\left(X_{1}, X_{4}\right)=(-2 A-2(p+q) B+1) g\left(X_{1}, X_{4}\right)
$$

and thus we obtain

$$
\operatorname{Ric}\left(X_{1}, X_{4}\right)=-\frac{-2 A-2(p+q) B+1}{2 B} g\left(X_{1}, X_{4}\right) .
$$

So, the manifold is Einstein. On the other hand by direct computation from (6) the scalar curvature is

$$
s c a l=\frac{(2 p+2 q)(2 p+2 q+1)}{4(p+q)+1} .
$$

This shows the scalar curvature is positive. Let choose $X_{1}=X_{4}, X_{2}=X_{3}$ unit and orthogonal vector fields in (5). Then the sectional curvature is obtained by

$$
k\left(X_{1}, X_{2}\right)=A+2(p+q) B-1=A=\frac{s c a l}{(2 p+2 q+1)(2 p+2 q)}
$$

Thus, the proof is completed.

An Einstein manifold is also Einstein under concircular transformation. Yano proved that a concircular flat Riemann manifold is Einstein [3]. By similar way we can easily obtain following result.

Theorem 8. A concircular flat normal metric contact pair manifold is Einstein.

Our finally result is about quasi-conformal flatness of normal metric contact pair manifold. 
Theorem 9. A quasi-conformally flat normal metric contact pair manifold;

(1) is an Einstein manifold with a positive scalar curvature

(2) is a space of constant curvature.

Proof. Let $M$ be a quasi-conformally flat normal metric contact pair manifold. Then for $X_{1}, X_{2}, X_{3}, X_{4} \in \Gamma(T M)$ we have

$$
\begin{aligned}
0= & a \mathcal{R}\left(X_{1}, X_{2}, X_{3}, X_{4}\right)+b\left[\operatorname{Ric}\left(X_{2}, X_{3}\right) g\left(X_{1}, X_{4}\right)\right. \\
& -\operatorname{Ric}\left(X_{1}, X_{3}\right) g\left(X_{2}, X_{4}\right) \\
& \left.+g\left(X_{2}, X_{3}\right) \operatorname{Ric}\left(X_{1}, X_{4}\right)-g\left(X_{1}, X_{3}\right) g\left(X_{2}, X_{4}\right)\right] \\
& -\frac{s c a l}{2 p+2 q+2}\left[\frac{a}{2 p+2 q+1}+2 b\right]\left[g\left(X_{2}, X_{3}\right) g\left(X_{1}, X_{4}\right)\right. \\
& \left.-g\left(X_{1}, X_{3}\right) g\left(X_{2}, X_{4}\right)\right] .
\end{aligned}
$$

Let write $\frac{s c a l}{2 p+2 q+2}\left[\frac{a}{2 p+2 q+1}+2 b\right]=K$ for brevity. In $[7]$, by taking $X_{1}=X_{4}=E_{i}$ and getting sum from $i=1$ to $i=2 p+2 q+2$ we obtain

$$
0=(a+b(2 p+2 q)) \operatorname{Ric}\left(X_{2}, X_{3}\right)+(b s c a l-K(2 p+2 q+1)) g\left(X_{2}, X_{3}\right)
$$

and therefore we get

$$
0=[a+b(2 p+2 q)]\left[\operatorname{Ric}\left(X_{2}, X_{3}\right)-\frac{s c a l}{2 p+2 q+2} g\left(X_{2}, X_{3}\right)\right] .
$$

Assume that $a+b(2 p+2 q) \neq 0$. Then we have

$$
\operatorname{Ric}\left(X_{2}, X_{3}\right)=\frac{s c a l}{2 p+2 q+2} g\left(X_{2}, X_{3}\right) .
$$

By taking $X_{2}=X_{3}=Z$ in (8) and from (3), we get positive scalar curvature as

$$
\text { scal }=2(p+q)(p+q+1) .
$$

So, the Ricci curvature has the following form:

$$
\operatorname{Ric}\left(X_{2}, X_{3}\right)=(p+q) g\left(X_{2}, X_{3}\right) .
$$

This shows manifold is Einstein.

On the other hand consider (9) in (7) we get

$$
\begin{aligned}
0= & a\left[\mathcal{R}\left(X_{1}, X_{2}, X_{3}, X_{4}\right)-\frac{p+q}{2 p+2 q+1}\left[g\left(X_{2}, X_{3}\right) g\left(X_{1}, X_{4}\right)\right.\right. \\
& \left.\left.-g\left(X_{1}, X_{3}\right) g\left(X_{2}, X_{4}\right)\right]\right] .
\end{aligned}
$$

If $a \neq 0$ we get

$$
\mathcal{R}\left(X_{1}, X_{2}, X_{3}, X_{4}\right)=\frac{p+q}{2 p+2 q+1}\left[g\left(X_{2}, X_{3}\right) g\left(X_{1}, X_{4}\right)-g\left(X_{1}, X_{3}\right) g\left(X_{2}, X_{4}\right)\right] .
$$

Thus the manifold is a space of constant curvature. 


\section{REFERENCES}

[1] Geiges, H., A brief history of contact geometry and topology, Expositiones Mathematicae, 19(1) (2001), 25-53.

[2] Kholodenko, A. L., Applications of contact geometry and topology in physics, World Scientific, (2013)

[3] Yano, K., Concircular geometry I: concircular transformations, Proceedings of the Imperial Academy, 16(6) (1940), 195-200.

[4] Yano, K., Sawaski, S., Riemannian manifolds admitting a conformal transformation group, J. Diff. Geo,. 2 (1968), 161-184

[5] De, U. C., Shaikh, A. A., Complex manifolds and contact manifolds, Narosa Publishing House, (2009).

[6] Turgut Vanli, A., Unal, I., Conformal, concircular, quasi-conformal and conharmonic flatness on normal complex contact metric manifolds. International Journal of Geometric Methods in Modern Physics, 14(05) (2017), 1750067.

[7] Blair, D. E., Ludden, G. D., Yano, K., Geometry of complex manifolds similar to the CalabiEckmann manifolds, Journal of Differential Geometry, 9(2) (1974), 263-274.

[8] Bande, G. and Hadjar, A., Contact pairs. Tohoku Mathematical Journal, Second Series, 57(2) (2005), 247-260.

[9] Bande, G., Hadjar, A., On normal contact pairs, International Journal of Mathematics, 21(06) (2010), 737-754.

[10] Bande, G., Hadjar, A., Contact pair structures and associated metrics, In Differential Geometry, (2009), 266-275

[11] Bande, G., Blair, D. E., Hadjar, A., On the curvature of metric contact pairs, Mediterranean journal of mathematics, 10(2) (2013), 989-1009.

[12] Bande, G., Blair, D. E., Hadjar, A., Bochner and conformal flatness of normal metric contact pairs, Annals of Global Analysis and Geometry, 48(1) (2015), 47-56.

[13] Blair, D. E., Riemannian geometry of contact and symplectic manifolds, Springer Science Business Media, (2010).

[14] Kobayashi, S., Remarks on complex contact manifolds, Proc. Amer. Math. Soc., 10 (1959), 164-167.

[15] Ishihara, S., Konishi, M., Complex almost contact structures in a complex contact manifold, Kodai Math. J., 5 (1982), 30-37

[16] Korkmaz, B., Normality of complex contact manifolds, Rocky Mountain J. Math., 30 (2000), $1343-1380$

[17] Turgut Vanli, A., Blair, D. E., The Boothby-Wang Fibration of the Iwasawa Manifold as a Critical Point of the Energy, Monatsh. Math. 147 (2006), 75-84.

[18] Bande, G., Hadjar, A., On the characteristic foliations of metric contact pairs, Harmonic Maps and Differential Geometry. Contemp. Math, 542 (2011), 255-259.

[19] Beldjilali, G., Belkhelfa, M., Structures on the product of two almost Hermitian almost contact manifolds, International Electronic Journal of Geometry, 9(2) (2016), 80-86. 